\title{
Neurofibromatatosis Type 1 in Association with Giant Congenital Melanocytic Naevus and Vitiligo

\author{
${ }^{* 1}$ Hamza Yildiz, ${ }^{2}$ Huseyin Saman \\ 2Department of Dermatology, Isparta Military Hospital, 32020, Isparta/Turkey \\ ${ }^{* 1}$ hamzayildiz@gmail.com,2drhsza@mynet.com
} \\ 1Department of Dermatology, Eskisehir Military Hospital, 26020, Eskisehir/Turkey
}

\begin{abstract}
Giant congenital melanocytic naevus and neurofibromatosis type 1 may rarely occur together. We reported here an unusual case with coexistent giant congenital melanocytic naevus, vitiligo and type 1 neurofibromatosis.
\end{abstract}

Keywords: Giant congenital melanocytic naevus, neurofibromatosis, vitiligo, malignant melanoma.

\section{INTRODUCTION}

It is well known various kinds of pigmented skin lesions are associated with neurofibromatosis (NF) such as café au lait, naevus spilus, naevus of Ota and giant congenital melanocytic naevus (GCMN) [1-3]. However, GCMN and vitiligo are rarely associated with NF. To the best of our knowledge, there is no such case report in English literature. Hereby we report the first case of GCMN and vitiligo in a patient with NF.

\section{Case Report}

A 22-year-old boy was referred to the dermatology outpatient clinic for evaluation of widespread dark patches and various pigmented lesions on his skin. Even though the various pigmented lesions were asymptomatic, these lesions bothered the patient cosmetically. He had no family history of such lesions. There were no associations with hair, nail or mucosal abnormalities. There was no history of musculoskeletal or neurological complaints. Systemic examination was normal. There was no bone defect. He has been a smoker for 10 years. He did not consume alcohol.

There were multiple café au lait macules and multiple, painless, firm, soft, skin-coloured nodules (neurofibromas) on his trunk, back and extremities (Figure 1A). The palms, soles and genitalia are spared. These café au lait macules and giant congenital melanocytic naevus are present since birth and increasing in both number and size. Axillary freckling developed during puberty.

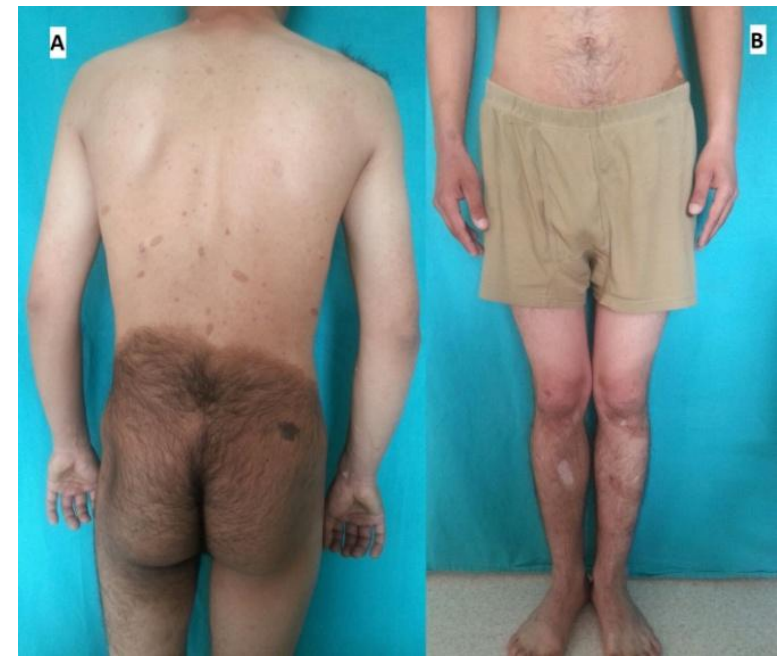

Figure 1 (A) The patient's large congenital nevus on his buttock and small congenital nevus on his right shoulder. (B) White macules on the bilateral pretibial area 
Examination revealed two hyperpigmented macular lesions measured around 24x23 (bilaterally distributed on his buttock) and 6x3 (on his right shoulder) $\mathrm{cm}$ in size with irregular borders (Figure 1A, 2C). Terminal hairs (hypertrichosis) were observed in the areas of hyperpigmentation. These lesions were present since birth and were progressively increasing in size. These macules were diagnosed with GCMN.

There were also white macules over the bilateral pretibial area of lower extremities (Figure 2A) and on the right wrist measured around $1.5 \times 1 \mathrm{~cm}$ (Figure 2B), but he was asymptomatic. These hypopigmented macules and patches were also flat and non-scaly. They had clear demarcation. The largest white macule on the lower extremities was measured about $3 \times 2 \mathrm{~cm}$. The hair over the macules was white (leukotrichia), dry and coarse (Figure 2A). A clinical diagnosis of vitiligo was made.

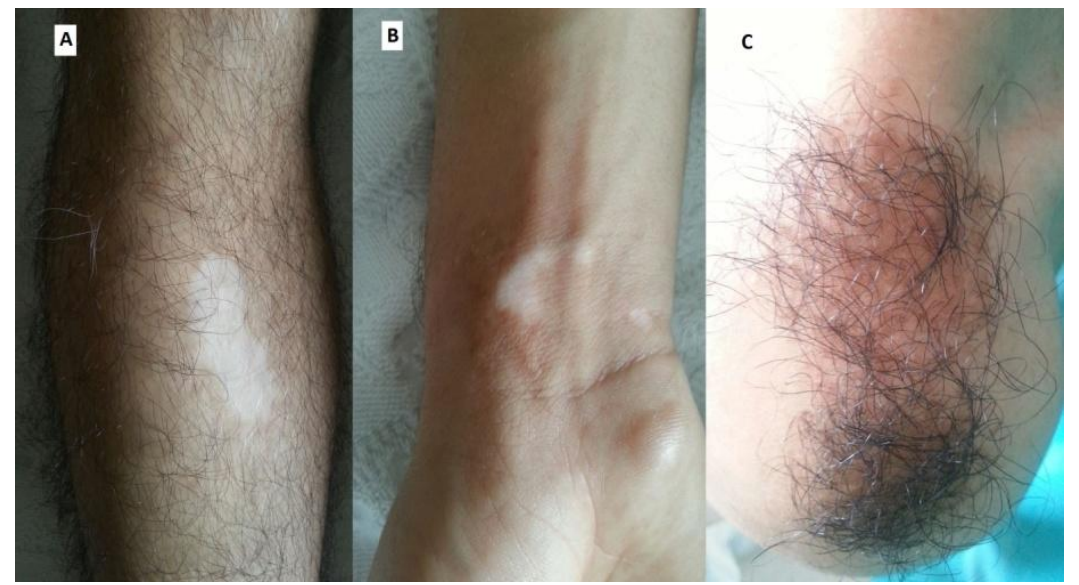

Figure 2 (A) White macules on the bilateral pretibial area of lower extremities and (B) on the right wrist. The hair over the macules was white (leukotrichia). (C) Congenital nevus on his right shoulder.

Slit-lamp examination revealed Lisch nodules. Neurological examination was normal. Laboratory investigation, including blood count, serum biochemistry, thyroid unction test, vitamin $\mathrm{B}_{12}$, folic acid and erythrocyte sedimentation rate revealed no abnormality. Skeletal X-ray, brain and spinal magnetic resonance imaging were normal.

Based on clinical findings, the patient was diagnosed to have a NF type 1 with coexisting GCMN and vitiligo. The patient was informed about the complications of $\mathrm{NF}$ and the need for regular follow up.

\section{DISCUSSION}

Congenital melanocytic naevus (CMN) is a benign neoplasm composed of nevomelanocytes. The giant form is a rare variant of $\mathrm{CMN}$ and rare conditions [1]. It is usually defined as a melanocytic lesion recognized at birth that will reach a diameter larger than $20 \mathrm{~cm}$ in adulthood, and its incidence is estimated in <1:20,000 newborns [2,3]. Despite its rarity, it may associate with severe abnormalities such as meningocele, spina bifida occulta, club foot and hypertrophy or atrophy of deeper structures of a limb, premature aging syndromes, vitiligo, Carney complex, neurofibroma, lipoma and dysplasia of bilateral hip impact on the patient [3].

Hadj et al. reported a case of GCMN and vitiligo with neurofibroma-like lesions. They stated that NF type 1 eliminated because no other clinical diagnostic criteria were present. They emphasized that the signification of vitiligo, which can testify of a possible malignant transformation of the GCMN to a melanoma, and highlights the importance of an accurate diagnosis and a close follow-up of such patient [1]. Our case is the first case of GCMN and vitiligo in a patient with NF.

Hadj et al. reported satellite nevi may be present in 74-91\% of the GMNs [1]. Our patient has satellite nevi on his right shoulder.

For a long time, a possible relationship between malignant melanoma and vitiligo has been argued. Some of the data published suggested an association between vitiligo, vitiligo-like leukoderma or halo nevi and the appearance of malignant melanoma [4]. The GCMN has a potential for transformation into malignancies, especially malignant melanoma [1]. Doherty et al. described a patient with segmental NF and large congenital nevus who developed malignant melanoma in the same anatomic distribution [5]. No malignant lesion was found with dermatolologic and dermatoscopic examination in this patient. 
In conclusion, the risk of melanoma in the patient with GCMN should be kept in mind, physicians should close clinical and histopathological follow-up their patients, especially if the patient has vitiligo, additionally.

Conflict of interest The authors declare that they have no conflict of interest.

\section{REFERENCES}

[1] Hadj I, Meziane M, Harmouch T, Mernissi FZ. Giant congenital malanocytic nevus with neurofibroma-like lesions and onset of vitiligo. J Dermatol \& Dermatol Sur 2015; 19(1):58-61.

[2] Viana AC, Gontijo B, Bittencourt FV. Giant congenital melanocytic naevus. An Bras Dermatol 2013; 88(1):190.

[3] Shang Z, Dai T, Ren Y. Neurofibroma and lipoma in association with giant congenital melanocytic nevus coexisting in one nodule: a case report. Int J Clin Exp Med 2015; 8(7):1104851.

[4] Grefer K, Hofmann M, Sterry W, Trefzer U. Coexistence of a congenital giant hairy nevus and extensive vitiligo. Dermatology 2003; 207(2):199-201.

[5] Doherty SD, George S, Prieto VG, Gershenwald JE, Duvic M. Segmental neurofibromatosis in association with a large congenital nevus and malignant melanoma. Dermatol Online J 2006; 12(7):22. 